\title{
Prognostic value of lymph node ratio in surgically treated cases of vulvar cancer: a tertiary care centre experience
}

\author{
Pabashi Poddar MS, M.Ch, Shilpa Patel, MD, Ruchi Arora, DGO, DNB, Chetana Parekh, DGO, DNB, \\ Pariseema Dave, MD, Sangeetha Amin, MS
}

Department of Gynecological Oncology, Gujarat Cancer \& Research Institute, Ahmedabad, India

\section{Objective}

This study aimed to study the patterns of recurrence in surgically treated cases of vulvar cancer (VC) and determine the factors associated with recurrence, with a special emphasis on lymph node ratio (LNR).

\section{Methods}

This retrospective study examined VC patients primarily treated with surgery at our institute from January 2005 to December 2015. Demographic data, clinical characteristics, surgicohistopathological data, adjuvant treatment, follow up, and recurrence site and treatment were studied.

\section{Results}

Among the 111 cases treated, a recurrence rate of $18.9 \%$ was noted. Recurrence was most commonly local (61.9\%). On univariate and multivariable analyses of clinicopathological parameters, an LNR $>20$ had the highest hazard ratio for recurrence.

Conclusion

LNR may provide useful prognostic information in VC patients with positive inguinal lymph node status.

Keywords: Vulvar cancer; Lymph node ratio; Recurrence; Radiotherapy

\section{Introduction}

Vulvar malignancies are rare, comprising around 3-5\% of all gynecological malignancies [1]. The incidence of vulvar cancer (VC) increases with age, peaking around the 7th decade [2]. Squamous cell carcinoma (SCC) remains the most common pathology in VCs, followed by melanoma, basal cell carcinoma, and adenocarcinoma.

Surgery is the treatment of choice for $\mathrm{VCs}$, while inguinal lymph node (LN) involvement is the most important prognostic factor $[3,4]$. Adjuvant radiotherapy (RT) is indicated in cases of close or involved surgical margins and LN involvement. Concurrent chemoradiotherapy in a neoadjuvant or curative or palliative setting may be recommended in locally advanced VCs.

Despite these treatment modalities, recurrence rates remain high at $12-37 \%$. Given the rarity of these tumors, there is limited information regarding the prognostic factors involved in recurrence and the treatment options available, especially since most of these women are aged $>60$ years and have significant comorbidities [5].

Several studies have focused on the clinical relevance of lymph node ratio (LNR) in solid tumor malignancies such as endometrial, cervical, and ovarian cancers. LNR is defined as the ratio of the number of metastatic LNs to the total number of removed LNs. This parameter incorporates not only

Received: 2019.09.03. Revised: 2019.12.10. Accepted: 2019.12.15. Corresponding author: Shilpa M Patel, MD, OBGY, Prof and HOD Department of Gynecological Oncology, Gujarat Cancer \& Research Institute, Asarwa, Ahmedabad, Gujarat 380016, India.

E-mail: drshilpamukesh@gmail.com

https://orcid.org/0000-0002-3090-5455

Articles published in Obstet Gynecol Sci are open-access, distributed under the terms of the Creative Commons Attribution Non-Commercial License (http://creativecommons org/licenses/by-nc/3.0/) which permits unrestricted non-commercial use, distribution, and reproduction in any medium, provided the original work is properly cited.

Copyright $\odot 2020$ Korean Society of Obstetrics and Gynecology 


\section{Obstetrics \& Gynecology Science}

Pabashi Poddar, et al. Prognostic value of lymph node ratio in vulvar cancers

the burden of nodal disease and cancer spread but also the extent and quality of surgical debulking of LNs [6]. This study has been done to study the role of LNR in relation to recurrence in VC patients.

This study aimed to study the patterns of recurrence in surgically treated cases of VC and determine the factors associated with recurrence with special emphasis on LNR.

\section{Material and methods}

This retrospective study included VC patients primarily treated with surgery at our institute from January 2005 to December 2015. Demographic data, clinical characteristics, surgicohistopathological data, adjuvant treatment, follow-up, and recurrence site and treatment were studied. For staging purposes, the 2009 International Federation of Gynecology and Obstetrics (FIGO) classification was used. Appropriate Institute Review Board approval (IRC/2019/P-101) was received for the study.

\section{Inclusion criteria}

All patients with vulvar SCC who underwent primary surgery at our institute were eligible. All patients with vulvar SCC and a depth of invasion $>1 \mathrm{~mm}$ or diameter $>2 \mathrm{~cm}$ fit for primary surgical resection were included. Wide radical local excision or radical vulvectomy was planned depending upon the focality, tumor size, adjacent leukoplakia, tumor location, and patient age. We attempted to attain a $10-\mathrm{mm}$ tumorfree margin during surgery for all patients. After surgery, adjuvant RT was chosen based upon the final histopathological examination report for cases of FIGO III/IV and a close surgical margin $(<8 \mathrm{~mm})$.

\section{Exclusion criteria}

Patients with VC and a histopathology other than SCC, those who underwent surgery at another center, and those receiving neoadjuvant/curative/palliative chemotherapy/RT were excluded. Patients with locally advanced VC who might require a diversion stoma or exenteration were referred for neoadjuvant/curative chemo/RT on an individual basis after a tumor board discussion. Patients with metastatic VC were referred for palliative chemotherapy.

Patients were stratified into 3 risk groups according to LNR $(0 \%, 0-20 \%$, or $>20 \%)$ as published previously in the report from Gynecologic Oncology Group (GOG) protocol 37 [7]. Disease-free survival (DFS) was calculated in months from the date of surgery to the last follow-up or date of first recurrence, overall survival (OS) was calculated in months from the date of surgery to the last follow-up or date of death.

The statistical analysis was performed using Epi Info ${ }^{\text {TM }}$ 7.2.2.2 (Centers for Disease Control and Prevention). A descriptive statistical analysis was performed to calculate the means and corresponding standard deviations. Test of pro-

Table 1. Distribution of demographic parameters, pathological data and stratification based on LNR of the patients

\begin{tabular}{|c|c|}
\hline Variables & Values $(n=111)$ \\
\hline \multicolumn{2}{|l|}{ Age (yr) } \\
\hline $40-49$ & $25(22.5)$ \\
\hline $50-79$ & $84(75.6)$ \\
\hline$\geq 80$ & $2(1.8)$ \\
\hline \multicolumn{2}{|l|}{ Menopausal status } \\
\hline Pre-menopausal & $14(12.6)$ \\
\hline Post-menopausal & $97(87.4)$ \\
\hline \multicolumn{2}{|l|}{ Grade } \\
\hline I & $81(73.0)$ \\
\hline$\|$ & $25(22.5)$ \\
\hline III & $5(4.5)$ \\
\hline \multicolumn{2}{|l|}{ Tumor focality } \\
\hline Multifocal & $59(53.2)$ \\
\hline Unifocal & $52(46.8)$ \\
\hline \multicolumn{2}{|l|}{ Tumor size } \\
\hline $2-4 \mathrm{~cm}$ & $12(10.81)$ \\
\hline $4-6 \mathrm{~cm}$ & $59(53.15)$ \\
\hline$>6 \mathrm{~cm}$ & $40(36.04)$ \\
\hline \multicolumn{2}{|l|}{ FIGO Stage 2009} \\
\hline IB & $58(52.25)$ \\
\hline$\|$ & $11(9.99)$ \\
\hline IIIA & $5(4.5)$ \\
\hline$\| I I B$ & $6(5.41)$ \\
\hline IIIC & $31(27.93)$ \\
\hline \multicolumn{2}{|l|}{ LNR Distribution } \\
\hline LNR 0\% & $70(63.06)$ \\
\hline LNR 0-20\% & $32(28.83)$ \\
\hline LNR > $20 \%$ & $9(8.11)$ \\
\hline
\end{tabular}

Values are presented as number (\%).

FIGO, International Federation of Gynecology and Obstetrics LNR, lymph node ratio. 


\section{Obstetrics \& Gynecology Science}

Vol. 63, No. 2, 2020

portion was used to find the standard normal deviate (Z) to compare the different proportions, and the chi-square test was performed to find the associations. Kaplan-Meir survival analysis, a non-parametric statistic, was used to estimate the survival function of the patients with different LNR. The log-rank test was used to compare the survival patterns of patients with different parameters. The hazard ratio (HR) was calculated to find the factors associated with recurrence after the adjustment of confounding factors. Values of $P<0.05$ were considered statistically significant.

\section{Results}

A total of 111 patients were included in the study. The mean patient age was 58.24 years (Table 1). Inguinal LNs were positive for metastasis in 42 cases (37.8\%) were FIGO stage III, while 31 cases (27.93\%) had perinodal extension (FIGO stage IIIC) (Table 1). There were 8.71 and 0.49 resected and

Table 2. Distribution of treatment and types of recurrence

\begin{tabular}{lc}
\hline Variables & Values \\
\hline Type of treatment & 111 \\
Observation & $50(45.0)$ \\
Adjuvant treatment received & $56(50.5)$ \\
Perineal radiotherapy & $55(49.5)$ \\
Inguinal radiotherapy & $37(33.3)$ \\
Only chemotherapy & $1(0.9)$ \\
No adjuvant received despite planning & 5 \\
Types of recurrence & 21 \\
Local & $13(61.9)$ \\
Groin & $3(14.3)$ \\
Pelvic nodal & $2(9.5)$ \\
Distal (Vertebral, 1; Lung, 1; Lung, 1) & $3(14.3)$ \\
\hline
\end{tabular}

Values are presented as number (\%). positive nodes on the right side versus 8.84 and 0.47 on the left side, respectively. Bilateral inguinal node positivity was present in 11 of 42 node-positive cases (26.2\%). Patients were stratified into 3 groups based on LNR values: $0 \%(n=70)$; $0-20 \%(n=32)$, and $>20 \%$ ( $n=9)$ (Table 1).

Of 111 cases, 50 were kept on observation and 61 were planned for adjuvant therapy. Of the 61 cases, 5 could not be treated with RT (residual disease with wound breakdown, poor RT tolerance due to old age [ $n=2]$, skin graft wound breakdown, non-healing ulcer in the groin). RT was delayed more than 8 weeks in 14 cases due to wound breakdown (Table 2). One patient received only chemotherapy due to microscopic metastases in the LN. All 55 patients received perineal RT, while 37 also received bilateral inguinal RT. On follow-up, lymphedema developed in 23 (20.7\%) cases and deep vein thrombosis in 4 cases.

At the median follow-up of 22.8 months, the mean OS was 27.8 months and DFS was 26 months. The mean time to recurrence was 12.2 months. A total of 21 cases recurred (18.9\%); of them, 17 (80.95\%) occurred within the first 2 years after surgery. Local recurrence was the most common type (61.9\% cases) (Table 2). Most cases of recurrence occurred in patients with Stage III disease (61.9\%) (Table 3). Recurrence was treated with radiation in 7 cases, wide local excision in 1 case, and chemotherapy in 13 cases. A stagewise distribution of recurrence is described in Table 4.

A further statistical analysis was performed of the factors responsible for recurrence (Table 5, Fig. 1). On univariate and

Table 3. Stage wise distribution of recurrence

\begin{tabular}{lccccc}
\hline Stage & Total & Local & Groin & Pelvic node & Distal \\
\hline Stage I & $7(33.3)$ & 6 & 1 & 0 & 0 \\
Stage II & $1(4.8)$ & 0 & 0 & 1 & 0 \\
Stage III & $13(61.9)$ & 7 & 2 & 1 & 3 \\
Total & 21 & 13 & 3 & 2 & 3 \\
\hline
\end{tabular}

Values are presented as number (\%).

Table 4. Stage wise distribution of various individual risk factors associated with recurrence

\begin{tabular}{lccccccccccc}
\hline Stage & Total & Prior RT & $\begin{array}{c}\text { Age } \\
(>\mathbf{6 0})\end{array}$ & Grade III & $\begin{array}{c}\text { BOR } \\
<\mathbf{1 0} \mathbf{~ m m}\end{array}$ & $\begin{array}{c}\text { Margin } \\
(<\mathbf{5 m})\end{array}$ & $\begin{array}{c}\text { DOI } \\
(>\mathbf{4 m m})\end{array}$ & $\begin{array}{c}\text { Size } \\
(>\mathbf{4} \mathbf{~ c m})\end{array}$ & LVE + & $\begin{array}{c}\text { LNR } \\
(>\mathbf{2 0} \%)\end{array}$ & $\begin{array}{c}\text { LNR } \\
(\mathbf{0}-\mathbf{2 0})\end{array}$ \\
\hline$I$ & 7 & 1 & 3 & 1 & 4 & 2 & 3 & 7 & 1 & 0 & 0 \\
$\|$ & 1 & 1 & 1 & 0 & 0 & 0 & 1 & 1 & 0 & 0 & 0 \\
$\mathrm{III}$ & 13 & 10 & 6 & 0 & 6 & 7 & 11 & 13 & 8 & 3 & 9
\end{tabular}

RT, radiotherapy; BOR, base of resection; DOI, depth of invasion; LVE, lympho-vascular emboli; LNR, lymph node ratio. 


\section{Obstetrics \& Gynecology Science}

Pabashi Poddar, et al. Prognostic value of lymph node ratio in vulvar cancers

multivariate analyses of clinicopathological parameters, LNR 1-20, LNR $>20$, and LN positivity (FIGO stage III) were statistically significant factors for recurrence. The highest HR was noted for LNR $>20$, followed by LNR 1-20, and LN positivity. Lymphovascular emboli was a factor for recurrence but not significant. Factors including age $>60$ years, Grade III disease, and a tumor-free margin $<5 \mathrm{~mm}$ were not associated with recurrence.

The log rank test showed that the recurrence-free survival rate of patients with an LNR of 0 was significantly higher

Table 5. Univariable and multivariable analysis for recurrence in vulvar cancer

\begin{tabular}{lccc}
\hline Factors & HR & $\mathbf{9 5 \%} \mathbf{C l}$ for HR & $\boldsymbol{P}$-value \\
\hline Univariable analysis & & & \\
Age (>60 yr) & 0.934 & $0.466-2.455$ & 0.746 \\
Grade III & 0.956 & $0.386-2.678$ & 0.742 \\
Margin (<5 mm) & 0.186 & $0.651-1.633$ & 0.834 \\
LVE & 1.611 & $0.624-2.862$ & 0.212 \\
LNR (1-20) & 1.883 & $1.184-6.582$ & $0.027^{\text {a) }}$ \\
LNR (>20) & 2.764 & $1.780-5.457$ & $0.038^{\text {a) }}$ \\
Lymph node positive & 1.418 & $1.069-1.863$ & $0.042^{\text {a) }}$ \\
Multi-variable analysis & & & \\
LVE & 1.599 & $0.581-2.400$ & 0.363 \\
LNR (1-20) & 1.784 & $1.095-6.486$ & $0.031^{\text {a) }}$ \\
LNR (>20) & 2.672 & $1.161-5.358$ & $0.042^{\text {a) }}$ \\
Lymph node positive & 1.168 & $1.035-1.495$ & $0.044^{\text {a) }}$ \\
\hline
\end{tabular}

$\mathrm{HR}$, hazard ratio; $\mathrm{Cl}$, confidence interval; LVE, lymphovascular emboli; LNR, lymph node ratio.

a) Statistically significant.

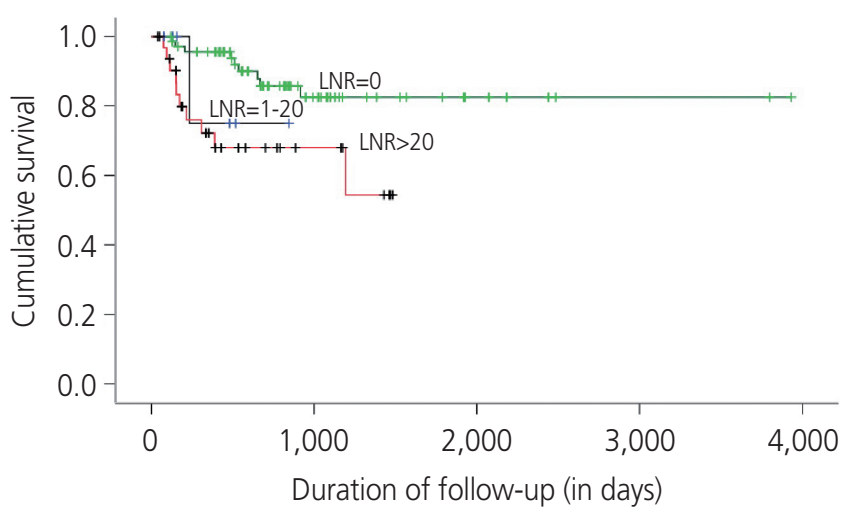

Fig. 1. Kaplan-Meier curves for LNR and recurrence-free survival. LNR, lymph node ratio. than that of patients with an LNR of 1-20 or $>20$ ( $\log$ rank=7.61; $P=0.02$ ). Similar results were noted on KaplanMeier analysis with the highest recurrence-free survival for an LNR of 0 (Fig. 1).

\section{Discussion}

VC is a less common gynecological cancer; surgery is the cornerstone of treatment [1]. Although nodal metastasis is the single most important predictor of prognosis, very few studies have determined the patterns of recurrence and factors other than nodal status that are causative of recurrence $[5,8,9]$.

Recurrence in VC is common and occurs in $12-37 \%$ of patients after initial treatment depending on tumor stage at the initial diagnosis; $40-80 \%$ of all recurrences occur within 2 years of the initial treatment. In a prospective study of 143 patients with vulvar SCC, Stehman et al. [7] found a median 35.9 months until local recurrence. The outcomes of patients with recurrence within 2 years after the initial surgery were worse than those of patients who had recurrences $>2$ years after the initial treatment. In our study, a total of 21 recurrences were noted $(18.9 \%)$ and the mean time to recurrence was 12.2 months; most recurrences were local and within the first 2 years after surgery (17 cases [80.95\%]).

More than half of VCs recur locally, presenting as isolated local recurrences or associated with the groin or distant recurrence. Risk factors for local recurrence include older age, larger tumor size, multifocal tumor, invasion depth $>2 \mathrm{~mm}$, lymphovascular space invasion, and the presence of $L N$ metastases at the initial treatment [10-12]. In our study, recurrences occurred primarily in the vulvar region (61.9\%), even in node-positive cases treated with adjuvant RT, suggesting that surgical excision with RT of the pelvic region could achieve local control in the groin but not the vulvar region. Further analysis of these aggressive node-positive tumors is required to shed light on their potential to cause recurrence.

In our study, a total of 9 recurrences were noted in patients with FIGO stages I-II (local, 6; groin, 1; pelvis, 1) even after adjuvant radiation in 2 cases. In another multicenter study by Van Beekhuizen et al. [13] on patients with negative inguinofemoral LNs, recurrence was related to poor differentiation and LNs $<9$. In addition, the role of human papilloma virus in local inguinal recurrence and in secondary recurrence must be emphasized to understand future screening and treat- 


\title{
Obstetrics \& Gynecology Science
}

\author{
Vol. 63, No. 2, 2020
}

ment options.

Although it is obvious that a positive tumor margin is associated with an increased local recurrence rate, the association between tumor-free margin width and local recurrence rate is less clear. Heaps et al. found that increasing tumorfree margins were associated with a decrease in the local recurrence rate in a group of 135 patients [14]. In patients with a tumor-free margin $<8 \mathrm{~mm}$, there was a $48 \%$ risk of local recurrence compared to a $0 \%$ local recurrence rate for patients with a tumor-free margin $>8 \mathrm{~mm}$. However, subsequent studies by De Hullu et al. [15] yielded varying results regarding the tumor-free margin and risk of local recurrence. However, it is important to note that both of the abovementioned studies did not exclude node-positive patients. Moreover, in most of these studies, the difference between true recurrence and de novo tumors was not considered. Future studies should investigate the optimal tumor-free margin to prevent local recurrence. Even in our study, a tumor-free margin was not associated with recurrence.

RT has played an important role in the management of patients with vulvar SCC in combination with radical local excision. RT alone or in combination with LN dissection is highly effective at preventing inguinal node recurrence in patients with vulvar SCC. Adjuvant groin and pelvic RT is the standard of care for node-positive vulvar SCC for patients with 2 or more involved LNs, extracapsular extension, or inadequate LN dissection based on GOG protocol $37[7,16]$.

Data from GOG and Arbeitgsgemeinschaft Gynäkologische Onkologie have shown that a higher number of LN metastases was associated with a shorter survival time. Analysis of GOG protocol 37 data showed that LNR can be used to stratify patients into 3 prognostic risk groups: patients with an LNR $>20 \%$ had an increased rate of contralateral positive LNs (53\% vs. $29 \%$ ) and increased rates of recurrence (58\% vs. $36 \%$ ) and cancer-related death ( $58 \%$ vs. $33 \%$ ) compared to patients with an LNR $\leq 20[7,17]$. In our study, similar results were noted. A high LNR (>20) was associated with extracapsular invasion (stage IIIC in 100\%), tumor size $>4 \mathrm{~cm}$ (in $100 \%)$, contralateral positive LNs (66.7\%), and the highest HR (2.672) for recurrence among the prognostic factors studied. A higher HR for recurrence was noted for $L N R>20$ than for LNR <20 among LN-positive patients. Moreover, lymphovascular emboli and no adjuvant treatment were found to be other factors for recurrence but these were not statistically significant. Age of the patient, positive base of resection, and histopathological grade were not associated with recurrence.

Polterauer et al. [18] also analyzed the value of LNR in a study of 745 cases of VC. A high LNR $>20$ was associated with a poor recurrence-free survival and OS and allowed stratification of patients into risk groups for adjuvant treatment. They found an added advantage of adjuvant treatment in patients with higher LNR and proposed that LNR instead of nodal positivity might be more useful in selecting appropriate candidates for adjuvant radiation therapy after VC surgery.

LNR provides useful prognostic information in patients with positive inguinal LNs in VC. LNR adds important information not only related to disease burden but also to quality of surgical debulking of LNs. LNR is influenced by surgical technique, anatomic circumstances and the quality and accuracy of the pathological analysis, reflecting an objective parameter in the assessment of the procedure's extent and completeness. LNR allows more accurate stratification of patients than number of positive nodes independent of other established prognostic parameters. LNR might be useful for stratifying patients into risk-groups for selecting appropriate candidates for adjuvant radiation and should be assessed within future clinical trials.

This study is limited by its retrospective single-center study design. However, the present results enable the identification of patients at higher risk of recurrence for whom more aggressive treatment or surveillance is necessary. We investigated the prognostic value of LNR and evaluated the patterns of recurrence. Studies evaluating the efficacy and effectiveness of different adjuvant treatments are warranted.

In conclusion, like other gynecologic malignancies, discerning the clinicopathologic patterns of patients with VC could help the stratification of these patients for adjuvant RT of the vulva. Regular follow-up is needed to prevent multiple local recurrence, especially for patients with high-risk prognostic factors. Recurrences must be managed strategically according to their patterns and the uses of prior adjuvant therapies. Further treatment attempts and constant attention are needed to improve the outcomes of VC patients.

\section{Conflict of interest}

No potential conflict of interest relevant to this article was reported. 


\section{Obstetrics \& Gynecology Science}

Pabashi Poddar, et al. Prognostic value of lymph node ratio in vulvar cancers

\section{Ethical approval}

The study was approved by the authors' Institutional Review Board (IRC/2019/P-101). Written informed consents were obtained.

\section{Patient consent}

The patients provided written informed consent for the publication and the use of their images.

\section{References}

1. de Martel C, Ferlay J, Franceschi S, Vignat J, Bray F, Forman $D$, et al. Global burden of cancers attributable to infections in 2008: a review and synthetic analysis. Lancet Oncol 2012;13:607-15.

2. Akerman G, Dussour C, Haddad B, Paniel BJ, Rouzier R. Epidemiology of vulvar intra-epithelial neoplasias. Gynecol Obstet Fertil 2007;35:1251-6.

3. Raspagliesi F, Hanozet F, Ditto A, Solima E, Zanaboni F, Vecchione $F$, et al. Clinical and pathological prognostic factors in squamous cell carcinoma of the vulva. Gynecol Oncol 2006;102:333-7.

4. Hopkins MP, Reid GC, Vettrano I, Morley GW. Squamous cell carcinoma of the vulva: prognostic factors influencing survival. Gynecol Oncol 1991;43:113-7.

5. Iacoponi S, Zapardiel I, Diestro MD, Hernandez A, De Santiago J. Prognostic factors associated with local recurrence in squamous cell carcinoma of the vulva. J Gynecol Oncol 2013;24:242-8.

6. Kunos C, Simpkins F, Gibbons H, Tian C, Homesley H. Radiation therapy compared with pelvic node resection for node-positive vulvar cancer: a randomized controlled trial. Obstet Gynecol 2009;114:537-46.

7. Stehman FB, Bundy BN, Ball H, Clarke-Pearson DL. Sites of failure and times to failure in carcinoma of the vulva treated conservatively: a Gynecologic Oncology Group study. Am J Obstet Gynecol 1996;174:1128-32.

8. Bogani G, Cromi A, Serati M, Uccella S, Donato VD,
Casarin J, et al. Predictors and patterns of local, region$\mathrm{al}$, and distant failure in squamous cellcarcinoma of the vulva. Am J Clin Oncol 2017;40:235-40.

9. Cormio G, Loizzi V, Carriero C, Cazzolla A, Putignano G, Selvaggi $L$. Groin recurrence in carcinoma of the vulva: management and outcome. Eur J Cancer Care (Engl) 2010;19:302-7.

10. Gonzalez Bosquet J, Magrina JF, Gaffey TA, Hernandez $J$, Webb MJ, Cliby WA, et al. Long-term survival and disease recurrence in patients with primary squamous cell carcinoma of the vulva. Gynecol Oncol 2005;97:828-33.

11. Maggino T, Landoni F, Sartori E, Zola P, Gadducci A, Alessi $C$, et al. Patterns of recurrence in patients with squamous cell carcinoma of the vulva. A multicenter CTF study. Cancer 2000;89:116-22.

12. Piura B, Masotina A, Murdoch J, Lopes A, Morgan P, Monaghan J. Recurrent squamous cell carcinoma of the vulva: a study of 73 cases. Gynecol Oncol 1993;48:189-95.

13. Van Beekhuizen HJ, Auzin M, van den Einden LC, de Hullu JA, van der Velden J, Wildhagen MF, et al. Lymph node count at inguinofemoral lymphadenectomy and groin recurrences in vulvar cancer. Int J Gynecol Cancer 2014;24:773-8.

14. Heaps JM, Fu YS, Montz FJ, Hacker NF, Berek JS. Surgical-pathologic variables predictive of local recurrence in squamous cell carcinoma of the vulva. Gynecol Oncol 1990;38:309-14.

15. De Hullu JA, Hollema H, Lolkema S, Boezen M, Boonstra $\mathrm{H}$, Burger MP, et al. Vulvar carcinoma. The price of less radical surgery. Cancer 2002;95:2331-8.

16. Mahner S, Jueckstock J, Hilpert F, Neuser P, Harter P, de Gregorio N, et al. Adjuvant therapy in lymph node-positive vulvar cancer: the AGO-CaRE-1 study. J Natl Cancer Inst 2015;107:dju426.

17. Woelber L, Eulenburg C, Choschzick M, Kruell A, Petersen $C$, Gieseking F, et al. Prognostic role of lymph node metastases in vulvar cancer and implications for adjuvant treatment. Int J Gynecol Cancer 2012;22:503-8.

18. Polterauer S, Schwameis R, Grimm C, Macuks R, lacoponi $S$, Zalewski $K$, et al. Prognostic value of lymph node ratio and number of positive inguinal nodes in patients with vulvar cancer. Gynecol Oncol 2017;147:92-7. 